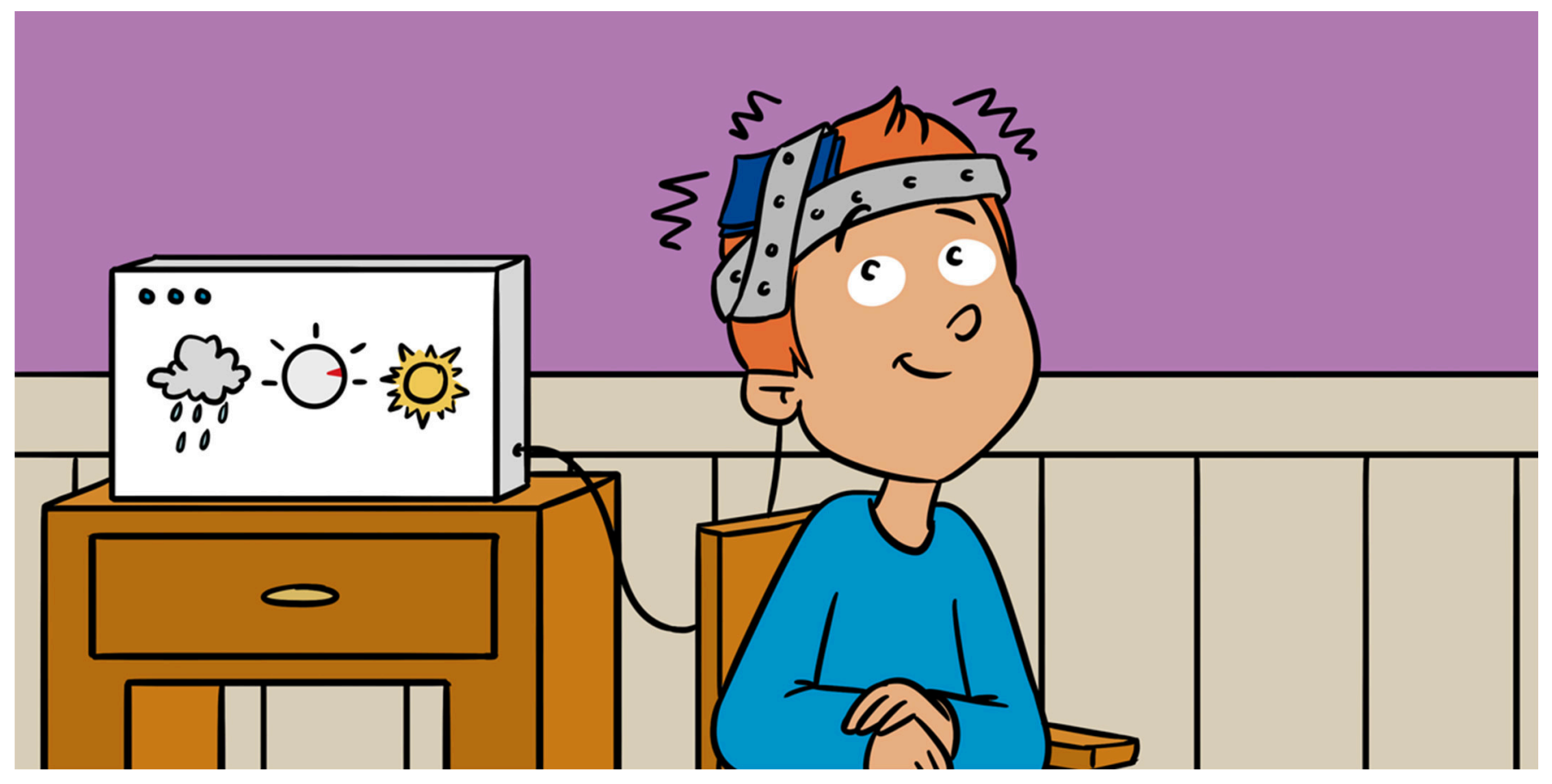

\title{
WAKE UP, BRAIN!: USING ELECTRICITY TO THINK AND FEEL DIFFERENTLY
}

\section{Mary A. Berg, Audrey M. Morrow and Michael C. Hout *}

Psychology Department, New Mexico State University, Las Cruces, NM, United States

YOUNG REVIEWERS:

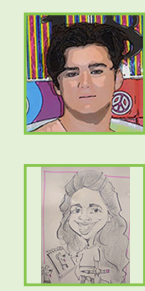

NICHOLAS

AGE: 15

SPANDANA

AGE: 10
Did you know that while reading this your brain is sending off chemical and electrical signals to help you to understand the words and their meaning? Your brain is made up of networks of small cells called neurons that communicate electrochemically to enable you to think, feel, and interact with the world around you. Because electrical charges are responsible for brain activity, electrical stimulation can in turn be used to change the brain's functioning. Brain stimulation has been used to treat mood disorders and stress, and it can even help people to solve problems, memorize information, and pay better attention. Luckily, many of the brain regions that control these functions are located in the cortex, which is the outer edge of the brain, closest to the skull. The cortex can be reached using a method called transcranial (into the skull) direct current stimulation (tDCS for short). In this article, we discuss how tDCS can be used to help people to think and feel differently. 


\section{NEURON}

Cells located in the brain that process and transmit information within the brain as well as between the brain and the body.

ION

An electrically charged particle.

ELECTROCHEMICAL Using both electrical and chemical energy.
Electrical brain stimulation can be a safe and effective way to temporarily alter brain activity without the need for brain surgery. Why would we want to alter brain activity? Well, people with brain damage or disorders can resolve potentially problematic patterns of brain activity through stimulation therapy. People with healthy brain functioning can also benefit from brain stimulation by improving their emotion regulation, attention, learning, problem solving, and memory abilities. You may be wondering how the brain can be stimulated without opening the skull. Electricity from the stimulation machine travels through your skull to affect the electrical signals responsible for brain activity. In this paper, we will discuss one of the most commonly used types of brain stimulation used to alter brain activity: transcranial direct current stimulation (tDCS).

\section{BRAIN CELLS USE ELECTRICITY AND CHEMICALS TO COMMUNICATE}

Neurons are cells in the brain. Neurons use both electrical charges and chemicals called ions to communicate with each other. We say that neurons have an electrochemical charge, and this charge changes, depending on whether the neuron is at rest or is sending a signal. Inside and between neurons there is fluid that contains ions, which are atoms or molecules that have a positive or negative charge. When a neuron is at rest, there are more negative ions inside and more positive ions outside of it (Figure 1A), giving the neuronal membrane a negative charge. When brain activity occurs, positive ions rush in through channels in the neuronal membrane (Figure 1B) and, when the charge gets high enough, the neuron sends a signal to communicate with nearby neurons (Figure $1 \mathrm{C}$ ). Think of this like a match that needs to be struck with just enough force; once it sparks, the whole match catches fire. Electrical stimulation on the scalp can target the electrochemical activity of the brain and alter the charges in neurons without the need for surgery. Because there is never only one neuron firing at a time, but rather "populations" of neurons that fire, altering the charge of the fluid surrounding neuronal populations can have significant effects on brain activity.

\section{HOW DOES TRANSCRANIAL DIRECT CURRENT STIMULATION AFFECT NEURONS?}

A group of neurons can be targeted with tDCS, which uses an electrical current to alter the electrochemical activity of a particular brain region. Two rubber electrodes are positioned on the head to target the brain area of interest, and these electrodes make an electrical circuit by sending current through the skin and the skull, affecting the brain underneath (Figure 2) [1]. 
Figure 1

(A) The electrochemical state of a neuron at rest compared with (B) a neuron that is sending a signal. Yellow circles represent negatively charged ions and pink circles represent positively charged ions At rest, there are more negative ions inside the cell and more positive ions outside the cell, which is why the neuron is said to have an overall negative voltage. When the neuron is activated, positive ions rush into the cell and negative ions rush out, making the voltage inside the cell more positive overall. (C) This change in charge then travels down the length of the cell to activate a neighboring cell.

\section{Figure 2}

\section{A possible tDCS} electrode set-up. In this example, the left DLPFC will receive negatively charged stimulation. As indicated by the arrows, the current flows from the tDCS machine through the red wire to the negative electrode. Then the current continues from the negative electrode to the positive electrode (via the person's skull and brain) and then back through the blue wire to the machine itself, creating a complete circuit. As a result of this current flow, the target neurons in the left DLPFC are less likely to send signals to other parts of the brain due to the negative charge in the brain near that electrode.
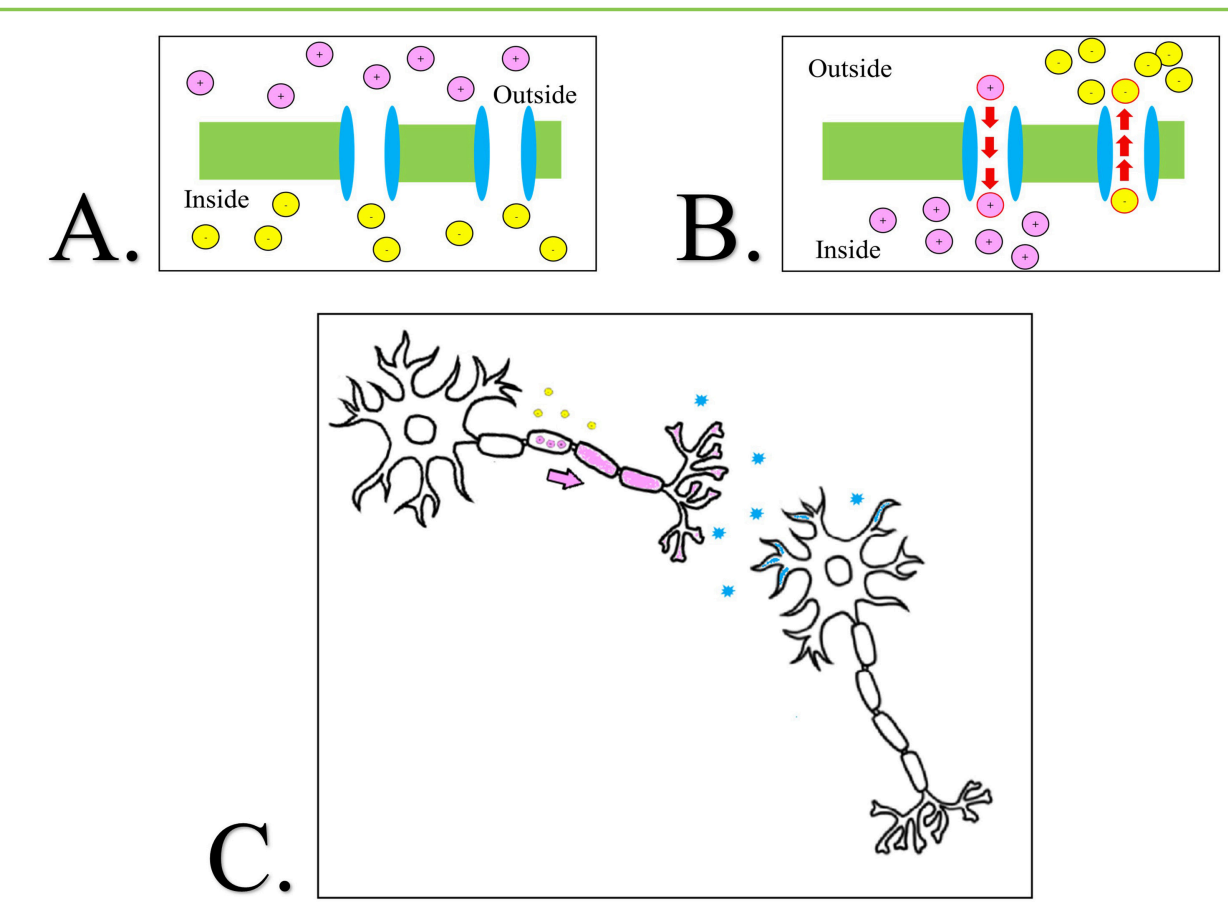

Figure 1

What are these electrical fields doing to alter neuronal activity? Because tDCS sends a current from one electrode to another in only one direction, stimulation creates a positive charge under one electrode and a negative charge under the other. These charges affect the neurons in different ways. Returning to the match analogy, different conditions might make the match more or less likely to 
catch fire, such as being in the hot sun vs. being in a damp climate. tDCS works by either increasing or decreasing the charge surrounding populations of neurons, making them more or less likely to send signals. If you were to line matches up, setting one on fire would set another on fire. Neurons communicate in a similar way, sending signals to neighboring neurons, which then send signals on to other neurons, and so on.

\section{WHAT CAN TDCS BE USED FOR?}

\section{Regulating Emotions}

A little sadness and stress is common in life, and most people experience these emotions in healthy amounts. However, prolonged periods of depression, anxiety, and stress can sometimes be indicators of mood-regulation disorders, which take a toll on mental (and physical) health. Ferrucci et al. [2] used tDCS to reduce the symptoms of one very common mood-regulation disorder, known as major depressive disorder (MDD). This disorder is associated with persistent feelings of sadness, low energy levels, changes in appetite, and loss of interest in activities. These symptoms are long lasting and thought to be caused by an imbalance of activity in the brain. Scans of the brain show lower-than-normal neuronal activity in an area in the front of the brain called the left dorsolateral prefrontal cortex (DLPFC; see Figure 3) and sometimes higher-than-normal neuronal activity in the right DLPFC. The DLPFC has links to emotion areas that are deeper in the brain, and thus plays an important role in controlling mood. In this study, participants with severe depression first completed questionnaires to assess their mood. Then, for 5 days in a row, participants underwent stimulation of the left DLPFC for $20 \mathrm{~min}$, twice each day. Participants completed the mood questionnaires again immediately after tDCS and most showed improvements on all of

Figure 3

Areas of the brain associated with various functions discussed in this article.

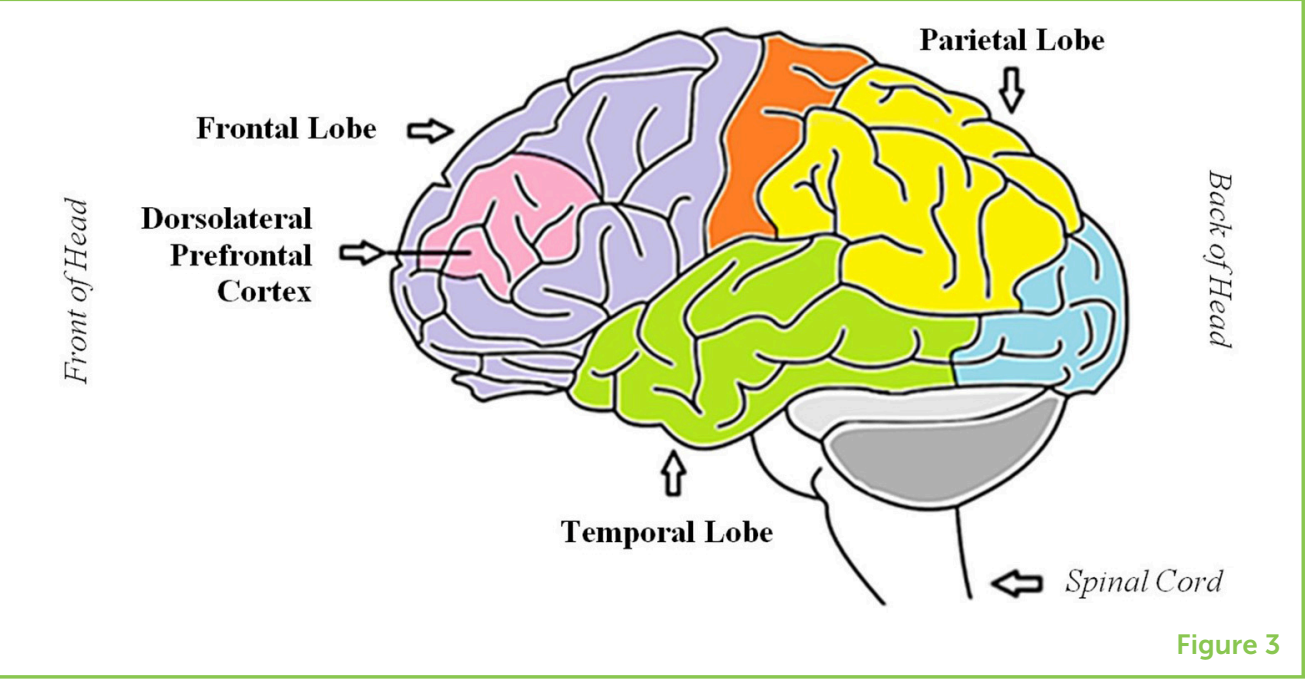




\section{COGNITION}

The mental processes by which we think, understand, pay attention, solve problems, remember information, and perceive the world around us them! Even when tested a month later, participants were still showing reduced symptoms of sadness and depression.

There are many other studies applying tDCS to the DLPFC to treat depression. However, Ferrucci's study is especially interesting in that all the patients were selected because they did not previously respond to anti-depressant medications. tDCS can best decrease the symptoms of depression when combined with medications and other forms of treatment, but it is interesting to know that stimulation alone can help, too.

\section{Improving cognitive function}

Cognition is the act of understanding the world around us through thought. This understanding requires a combination of attention, learning and problem solving, and memory. Different cognitive processes rely on different parts of the brain, and brain stimulation can be applied to different areas to encourage and enhance various aspects of cognition. Let us talk about a few specific cognitive functions that tDCS has been shown to influence.

\section{Attention}

Along with playing a role in mood regulation, the DLPFC is important for attention and impulses. The DLPFC is less active in people with a condition called attention deficit hyperactivity disorder (ADHD). ADHD makes it difficult to concentrate on one item or task at a time, ignore distractions and control impulsive behavior. However, a study of 37 patients with ADHD showed that stimulating the DLPFC using tDCS enhanced their ability to focus attention. Participants performed a set of tasks in which they had to respond to only some of the arrows or letters presented on a screen, while ignoring others. After tDCS, participants were less likely to respond to images they were supposed to ignore, which showed that they could control their behaviors more effectively [3].

\section{Learning}

Learning and problem solving are important cognitive processes that can be enhanced through brain stimulation. The area of the brain that must be stimulated to enhance these processes depends on what and how a person is learning. For example, researchers know that when we mentally manipulate numbers, the right parietal lobe of the brain is active (see Figure 3 for the location of the parietal lobe). Knowing this, researchers [4] applied tDCS to the right parietal lobe of healthy adults while those adults were learning the relationships between random symbols and their assigned numerical values. While learning about the fake number system, a third of participants received positive charge to the right parietal lobe, a third received negative charge to the right parietal lobe, and a third received fake (or "sham") stimulation to the right parietal lobe. After the participants completed the learning phase, they were tested on their newly acquired numerical 
knowledge. The results of this study showed that participants who received excitatory stimulation demonstrated a better understanding of the fake number system and acquired this understanding faster than the inhibitory and fake stimulation groups. Additionally, researchers followed up after 6 months and found that what they learned about the fake number system persisted over time! Based on this study, we know that excitatory tDCS can enhance our numerical learning abilities when applied to the right parietal lobe while the learning is taking place [4].

\section{Problem Solving}

Brain stimulation can also enhance problem-solving abilities. A group of researchers [5] investigated whether they could use brain stimulation to make participants better at solving problems. The researchers stimulated the brain in two places: the right anterior temporal lobe (rATL) and the left anterior temporal lobe (IATL) (see Figure 3 for the location of the temporal lobe). The temporal lobes are associated with thinking out-of-the-box and systematic problem solving. One group of participants received positive charge to the rATL and negative charge to the IATL. Another group received the reverse: negative charge to the rATL and positive charge to the IATL. A third group received fake stimulation. First, the participants were trained to solve a problem using a specific strategy. Then, after receiving $5 \mathrm{~min}$ of tDCS, they were asked to solve a similar problem that required thinking out-of-the-box. Sixty percent of participants who received positive charge to the rATL and negative charge to the IATL were able to successfully solve the second version of the problem before their time was up. Only $20 \%$ of the participants who received the reverse were able to solve the problem, which was about the same percentage of people from the fake condition that solved it. This study is a clear example of how tDCS can improve the ability to solve problems [5].

\section{Memory}

When you meet a new friend, you first learn his or her name. Over the next few interactions with that person, you often have to keep reminding yourself of that person's name until you have it memorized. This type of memory for factual knowledge is called declarative memory. When we are sleeping, our brains solidify the new declarative memories we learned during the day into our long-term memory. Researchers in Germany showed that applying tDCS to the sleeping brain can enhance declarative memory in healthy adults. Before stimulation, participants in this study learned 46 words pairs (i.e., bird-wing). After learning the word pairs, some of the participants went to sleep, while others stayed awake and watched a video. Half of the sleeping participants received excitatory stimulation to their frontal lobes and half received fake stimulation (and likewise with the awake participants). Upon waking up, the sleep condition participants took a recall test to see how well they remembered the 
word pairs from earlier. The wake condition did the same after they finished watching the video. The results showed that participants who received excitatory stimulation while they were sleeping were able to remember more word pairs compared with those in the wake condition, and also compared with the group that received fake stimulation! Excitatory stimulation did not help the participants in the wake condition to do any better on the recall test than those who received fake stimulation [6]. This experiment not only demonstrates the importance of getting a good night's sleep, but also the ability of brain stimulation to enhance memory.

\section{WHY IS THIS RESEARCH IMPORTANT?}

The research discussed in this article shows that tDCS can be useful for improving a variety of brain functions, including regulating emotions and enhancing cognitive functions, such as attention, learning, problem solving, and memory. But this important research also reminds us how much more there is to learn. Brain stimulation has the potential to positively impact the way we think and feel, but tDCS can currently only access the outer layers of the brain. Because of that, there are still several brain functions that have not been explored with this technology. Technology may advance in the future, penetrating deeper brain structures that lie below the cortex. The use of tDCS will likely continue to grow, so keep an eye out for exciting new findings in brain stimulation!

\section{REFERENCES}

1. Paulus, W. 2011. Transcranial electrical stimulation (tES-tDCS; tRNS, tACS) methods. Neuropsychol. Rehabil. 21:602-17. doi: 10.1080/09602011.2011.557292

2. Ferrucci, R., Bortolomasi, M., Vergari, M., Tadini, L., Salvoro, B., Giacopuzzi, M., et al. 2009. Transcranial direct current stimulation in severe, drug-resistant major depression. J. Affect. Disord. 118:215-9. doi: 10.1016/j.jad.2009.02.015

3. Allenby, C., Falcone, M., Bernardo, L., Wileyto, E. P., Rostain, A., Ramsay, J. R., et al. 2018. Transcranial direct current brain stimulation decreases impulsivity in ADHD. Brain Stimul. 11:974-81. doi: 10.1016/j.brs.2018.04.016

4. Hauser, T. U., Rotzer, S., Grabner, R. H., Mérillat, S., and Jäncke, L. 2013. Enhancing performance in numerical magnitude processing and mental arithmetic using transcranial Direct Current Stimulation (tDCS). Front. Hum. Neurosci. 7:244. doi: 10.3389/fnhum.2013.00244

5. Chi, R. P., and Snyder, A. W. 2011. Facilitate insight by non-invasive brain stimulation. PLOS ONE. 6:e16655. doi: 10.1371/journal.pone.0016655

6. Marshall, L., Mölle, M., Hallschmid, M., and Born, J. 2004. Transcranial direct current stimulation during sleep improves declarative memory. J. Neurosci. 24:9985-92. doi: 10.1523/JNEUROSCI.2725-04.2004 
SUBMITTED: 05 June 2018; ACCEPTED: 18 April 2019;

PUBLISHED ONLINE: 15 May 2019.

EDITED BY: Kathleen Y. Haaland, University of New Mexico, United States

CITATION: Berg MA, Morrow AM and Hout MC (2019) Wake Up, Brain!: Using Electricity to Think and Feel Differently. Front. Young Minds 7:62. doi: 10.3389/ frym.2019.00062

CONFLICT OF INTEREST STATEMENT: The authors declare that the research was conducted in the absence of any commercial or financial relationships that could be construed as a potential conflict of interest.

COPYRIGHT () 2019 Berg, Morrow and Hout. This is an open-access article distributed under the terms of the Creative Commons Attribution License (CC BY). The use, distribution or reproduction in other forums is permitted, provided the original author(s) and the copyright owner(s) are credited and that the original publication in this journal is cited, in accordance with accepted academic practice. No use, distribution or reproduction is permitted which does not comply with these terms.

\section{YOUNG REVIEWERS}

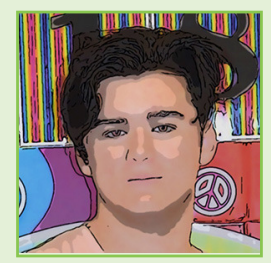

\section{NICHOLAS, AGE: 15}

I am interested heavily in all sciences, and am currently pursuing a career involved in either clinical medicine or medicinal research. I am a social person, and I love to walk and ride through forestries, camp and appreciate nature.

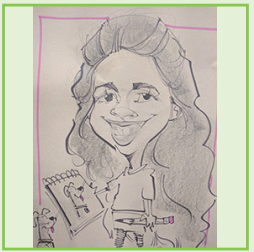

\section{SPANDANA, AGE: 10}

My name is Spandana. My favorite subject in school is science. My best friend is imagination and I love to write stories. Some of my hobbies are, talking, watching T.V., playing volleyball, and drawing. I love animals but favorite are dogs. I like to ask questions. My favorite colors are teal and purple.

\section{AUTHORS}

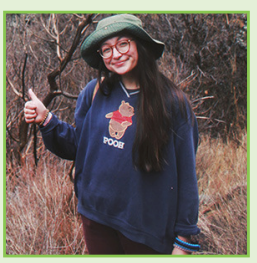

\section{MARY A. BERG}

Mary Berg attended college at South Dakota State University, where she received her Bachelor's degree in Psychology. She received her Master's degree in psychology at New Mexico State University, where she is currently finishing her studies in cognitive neuroscience. In completing her degree, she researched the effects of brain stimulation on basic spatial processing. Additionally, she uses electroencephalography (EEG) to compare brain waves of elderly people who are at high risk of falling vs. those at low risk. Mary's hobbies include photography, hiking, and playing with animals. 


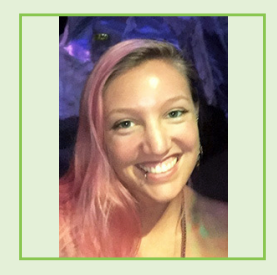

\section{AUDREY M. MORROW}

Audrey graduated with her Bachelor's degree in psychology from Towson University and then worked as a counselor for individuals with severe mood disorders and as a neuroscience research technician at the National Institutes on Aging. These experiences created a passion for understanding the brain in a way that can help healthy and clinical populations. Audrey went on to earn a Master's degree from New Mexico State University where her thesis research used brain stimulation to try to reduce the negative effects that stress has on cognition.

\section{MICHAEL C. HOUT}

I am an Associate Professor in the Psychology Department at New Mexico State University, and an Associate Editor at the journal Attention, Perception, \& Psychophysics. My research examines many different things, but I primarily study visual search (how people find things) and eye movements (where and why we move our eyes). In my limited spare time, I like to play with my dogs, go on motorcycle rides, hike, travel, and play hockey. *mhoutanmsu.edu 